\title{
BMJ Open Goal-directed fluid therapy in urgent GAstrointestinal Surgery - study protocol for A Randomised multicentre Trial: The GAS-ART trial
}

\author{
Anders Winther Voldby, ${ }^{1}$ Anne Albers Aaen, ${ }^{2}$ Ann Merete Møller, ${ }^{3}$ \\ Birgitte Brandstrup ${ }^{1}$
}

To cite: Voldby AW, Aaen AA, Møller AM, et al. Goaldirected fluid therapy in urgent GAstrointestinal Surgery—study protocol for A Randomised multicentre Trial: The GAS-ART trial. BMJ Open 2018;8:e022651. doi:10.1136/ bmjopen-2018-022651

- Prepublication history for this paper is available online. To view these files, please visit the journal online (http://dx.doi. org/10.1136/bmjopen-2018022651).

Received 28 February 2018 Revised 5 July 2018

Accepted 12 September 2018

Check for updates

(c) Author(s) (or their employer(s)) 2018. Re-use permitted under CC BY-NC. No commercial re-use. See rights and permissions. Published by BMJ.

${ }^{1}$ Department of Surgery, Holbaek University Hospital, Holbaek,

Denmark

${ }^{2}$ Department of Anaesthesiology, Holbaek University Hospital,

Holbaek, Denmark

${ }^{3}$ Department of Anaesthesiology and Intensive Care Medicine, Herlev University Hospital, Holbaek, Denmark

Correspondence to Dr Anders Winther Voldby; anwv@regionsjaelland.dk

\section{ABSTRACT}

Introduction Intravenous fluid therapy during gastrointestinal surgery is a life-saving part of the perioperative care. Too little fluid may lead to hypovolaemia, decreased organ perfusion and circulatory shock. Excessive fluid administration increases postoperative complications, worsens pulmonary and cardiac function as well as the healing of surgical wounds. Intraoperative individualised goal-directed fluid therapy (GDT) and zero-balance therapy (weight adjusted) has shown to reduce postoperative complications in elective surgery, but studies in urgent gastrointestinal surgery are sparse. The aim of the trial is to test whether zerobalance GDT reduces postoperative mortality and major complications following urgent surgery for obstructive bowel disease or perforation of the gastrointestinal tract compared with a protocolled standard of care.

Methods/analysis This study is a multicentre, randomised controlled trial with planned inclusion of 310 patients. The randomisation procedure is stratified by hospital and by obstructive bowel disease and perforation of the gastrointestinal tract. Patients are allocated into either 'the standard group' or 'the zero-balance GDT group'. The latter receive intraoperative GDT (guided by a stroke volume algorithm) and postoperative zero-balance fluid therapy based on body weight and fluid charts. The protocolled treatment continues until free oral intake or the seventh postoperative day. The primary composite outcome is death, unplanned reoperations, life-threatening thromboembolic and bleeding complications, a need for mechanical ventilation or dialysis. Secondary outcomes are additional complications, length of hospital stay, length of stay in the intensive care unit, length of mechanical ventilation, readmissions and time to death. Follow-up is 90 days. We plan intention-to-treat analysis of the primary outcome.

Ethics and dissemination The Danish Scientific Ethics Committee approved the GAS-ART trial before patient enrolment (J: SJ-436). Enrolment of patients began in August 2015 and is proceeding. We expect to publish the GAS-ART results in Summer 2019.

Trial registration number EudraCT 2015-000563-14.

\section{INTRODUCTION}

Death and complications are frequent following urgent major gastrointestinal
Strengths and limitations of this study

- This is a randomised controlled multicentre trial testing the effect of goal-directed fluid therapy in urgent gastrointestinal surgery.

- The multicentre design and an easy identification of the patient group supports broad clinical implementation of the study results.

- The primary outcome is clearly defined, clinically relevant and applies to the patients.

- Protocol adherence is secured by prelaminar and continuous teaching combined with regular assessment by the 'units of Good Clinical Practice', an independent data monitoring committee reinforcing the ICH-GCP (International Council for HarmonisationGood Clinical Practice) guideline.

Despite a set-up that is not blinded, the primary outcome will be assessor blinded.

surgery. ${ }^{1}$ Mortality rates are $15 \%$ to $25 \%,{ }^{2-4}$ and the morbidity is ominous with complication rates reported for more than $50 \%$ of the patients. ${ }^{5}$ Approximately 4500 patients annually undergo urgent gastrointestinal surgery in Denmark and several hundred of thousand people around the globe. ${ }^{67}$ Intravenous fluid administration is a life-saving part of the perioperative treatment. The challenge is to determine the right volume of fluid to be given. Within planned gastrointestinal surgery, the fluid volume given has noticeably influenced the postoperative outcome. ${ }^{8-13}$ However, trials testing perioperative fluid therapy for patients undergoing urgent surgery are sparse.

Intravenous fluid administration is necessary for upholding circulation and securing oxygen delivery to vital organs. Patients suffering from conditions requiring an urgent surgical intervention are frequently impaired by compromised fluid intake, nausea, vomiting, sepsis and other pathological fluid losses highlighting the vital 
need for intravenous fluid therapy to prevent circulatory shock and death. Thus, liberal intravenous fluid administration in volumes far exceeding the losses before, during and after the surgical procedure is common practice. ${ }^{14} 15$ On the other hand, there is no reason to believe that the harmful effect of fluid overload seen in studies of elective surgical patients is not valid for the patients undergoing urgent surgery. Interstitial oedema aggravate tissue inflammation, compromises wound healing and promotes anastomosis leakage. ${ }^{81016} 17$ In addition, cardiac arrhythmia, pulmonary oedema and acute respiratory distress syndrome may be the result.

The circulatory volume is difficult to measure or even estimate based on standard surveillance. Acute inflammation and stress response affect heart rate (HR) and diuresis. Arterial blood pressure and central vein pressure (CVP) may react on severe hypovolaemia, but does not reliably estimate normovolaemia or fluid overload, that is, does not tell when to stop the intravenous fluid infusion. Accordingly, the common physiological parameters are incapable of guiding clinical fluid therapy. As the patients are often hypovolaemic at admission, a simple in-out fluid balance (as used in trials of elective surgery to avoid fluid overload) is not useful for the urgent surgical patients.

The call for a more dynamic variable has led to the use of stroke volume (SV) to guide the fluid administration, the so-called goal-directed fluid therapy (GDT). Aiming at a submaximal SV using bolus infusions of a colloid, GDT possesses the ability to avoid both hypovolaemia as well as excessive fluid administration. Studies, using intraoperative GDT in planned gastrointestinal surgery, have shown to reduce length of hospital stay and complications. ${ }^{12} 1318-21$

The presented trial is testing whether an intervention using intraoperative GDT followed by a postoperative zero balance, named the 'zero-balance GDT' strategy (the GDT group), reduces postoperative mortality and major complications following urgent surgery of obstructive bowel disease or gastrointestinal perforation compared with a standard group (SDT group). The STD group follows an algorithm for fluid therapy used in the PULP trial (Peptic ULcer Perforation) ${ }^{22}$ resembling the fluid strategy used in River's study of patients with sepsis. ${ }^{23} \mathrm{~A}$ fluid strategy, that to our knowledge is the best evidencebased fluid algorithm used in the urgent setting. This treatment is based on mean arterial pressure (MAP), diuresis, HR, CVP and central venous oxygen saturation $\left(\mathrm{S}_{\mathrm{CV}} \mathrm{O}_{2}\right)$. We suggest that zero-balance GDT may hold the ability to detect both hypovolaemia and fluid overload and guide the fluid therapy towards normovolaemia, thus reducing complications and death following urgent surgery.

The hypotheses are the following:

- Hypovolaemia as well as fluid overload compromises cardiac and pulmonary function prompting postoperative complications such as arrhythmia, myocardial infarcts, other thromboembolic events, pneumonia, pulmonary congestion, exudation in the pleural cavity, pulmonary oedema and acute respiratory distress syndrome.

- Hypovolaemia as well as fluid overload is harmful for the healing of tissues and surgical wounds, causing complications related to poor tissue healing and infection, that is, wound infections, wound rupture (superficial and deep), anastomosis leakage and separation of a stoma from the skin.

\section{METHODS AND DESIGN}

The trial is a randomised, parallel group, open-label, multicentre, superiority trial. The primary outcome will be assessor blinded. The study protocol adheres to the SPIRIT (Standard Protocol Items: Recommendations for Interventional Trials) reporting guidelines. ${ }^{24}$ We launched the study at five public university hospitals from the three most Eastern regions in Denmark handling urgent surgery from 1.5 million inhabitants. In Denmark, public hospitals are free of charge and treat all patients in the need for urgent surgery.

\section{Eligibility criteria}

Anaesthesiologists and surgeons collaborate to carry out the trial. Selected trial anaesthetists are responsible for conducting the intraoperative GDT intervention.

\section{Inclusion criteria}

- The need for urgent gastrointestinal surgery due to radiologically verified gastrointestinal perforation or obstructive bowel disease.

- The presence of an anaesthetist qualified of conducting the intraoperative GDT intervention.

\section{Exclusion criteria}

- Surgery of palliative purposes and the dying patient (American Society of Anaesthesiologist (ASA) group 5 to 6 -ASA physical status classification).

- Major intra-abdominal surgery within the last 30 days.

- Iatrogenic gastrointestinal perforation.

- Dialysis on a regular basis.

- Patients unable to give informed consent for any reason.

- Age younger than 18 years.

- Pregnancy (positive urinary human chorionic gonadotropin).

\section{Intervention}

The patients are allocated to either (1) the GDT group or (2) the STD group. The treatment is initiated alongside the induction of the anaesthesia. Monitoring and data collection continue until the patient can drink and eat freely or to the seventh postoperative day.

In both groups, the treating physician administers preoperative antibiotics, antithrombotic and analgesia according to regional guidelines. The perioperative goals are MAP $>65 \mathrm{~mm} \mathrm{Hg}, \mathrm{HR}<100$ beats $/ \mathrm{min}$ and $\mathrm{SaO}_{2}$ (arterial oxygen saturation) $>94 \%$. Induction of anaesthesia is by propofol. Fentanyl and rocuronium are used if needed. Anaesthesia is by sevoflurane and fentanyl or propofol 


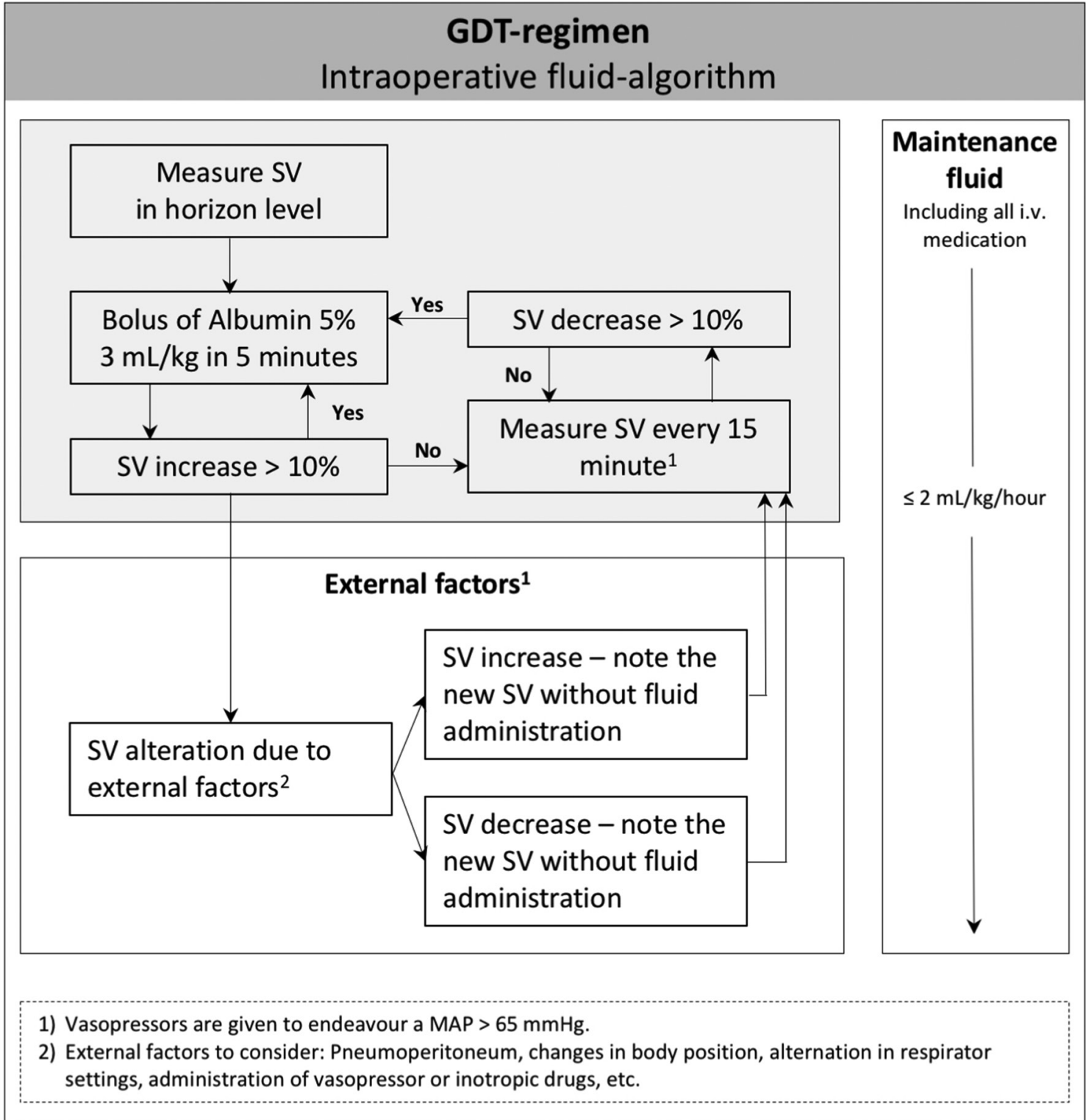

Figure 1 GDT regimen. Intraoperative fluid algorithm. GDT, goal-directed fluid therapy; i.v., intravenous; MAP, mean arterial pressure; SV, stroke volume.

and remifentanil. We allow local divergences. Additional epidural analgesia is used for laparotomy. We use invasive blood pressure monitoring in both groups and will keep haemoglobin $>70 \mathrm{~g} / \mathrm{L}(4.3 \mathrm{mmol} / \mathrm{L}) \quad($ or $>80 \mathrm{~g} / \mathrm{L}$ $(5.0 \mathrm{mmol} / \mathrm{L})$ for patients with chronic ischaemic heart disease and $>90 \mathrm{~g} / \mathrm{L}(5.6 \mathrm{mmol} / \mathrm{L})$ in case of acute ischaemic heart disease) using blood replacement therapy. Critical blood loss is treated with replacement of blood, plasma and platelets in the ratio 3:3:1. Intraoperatively, hourly diuresis is registered and arterial blood samples analysed.

In the GDT group, fluid optimisation follows the SV algorithm seen in figure 1, which ends when the patient leaves the operating room and proceeds as zero-balance approach. We use the FloTrac sensor and the EV1000 monitor from Edwards Lifesciences. Before surgery, SV is measured and consecutive boluses of $3 \mathrm{~mL} / \mathrm{kg}$ albumin
$5 \%$ are given until increase in SV is below $10 \%$. Then, SV is measured every 15 minutes. The procedure is repeated if SV decreases $>10 \%$. Changes in SV following external factors, such as pneumoperitoneum, changes in body position, alternation in respirator settings or administration of vasopressor or inotropic drugs, do not require fluid administration. Additional maintenance fluid replaces only pathological and physiological loses in the ratio $1: 1$, the latter amounting no more than $2 \mathrm{~mL} / \mathrm{kg} /$ hour including all intravenous medication (antibiotics, anaesthetics, etc). The aim is a zero-balance, limiting unnecessary interstitial fluid accumulation. Additional vasopressors are given to endeavour a MAP $>65 \mathrm{~mm} \mathrm{Hg}$.

Postoperative fluid administration is adjusted according to the fluid chart and the postoperative body weight as a zero-balance approach. Furosemide is given if the accumulated fluid balance exceeds $2 \mathrm{~L}$ or the body weight 


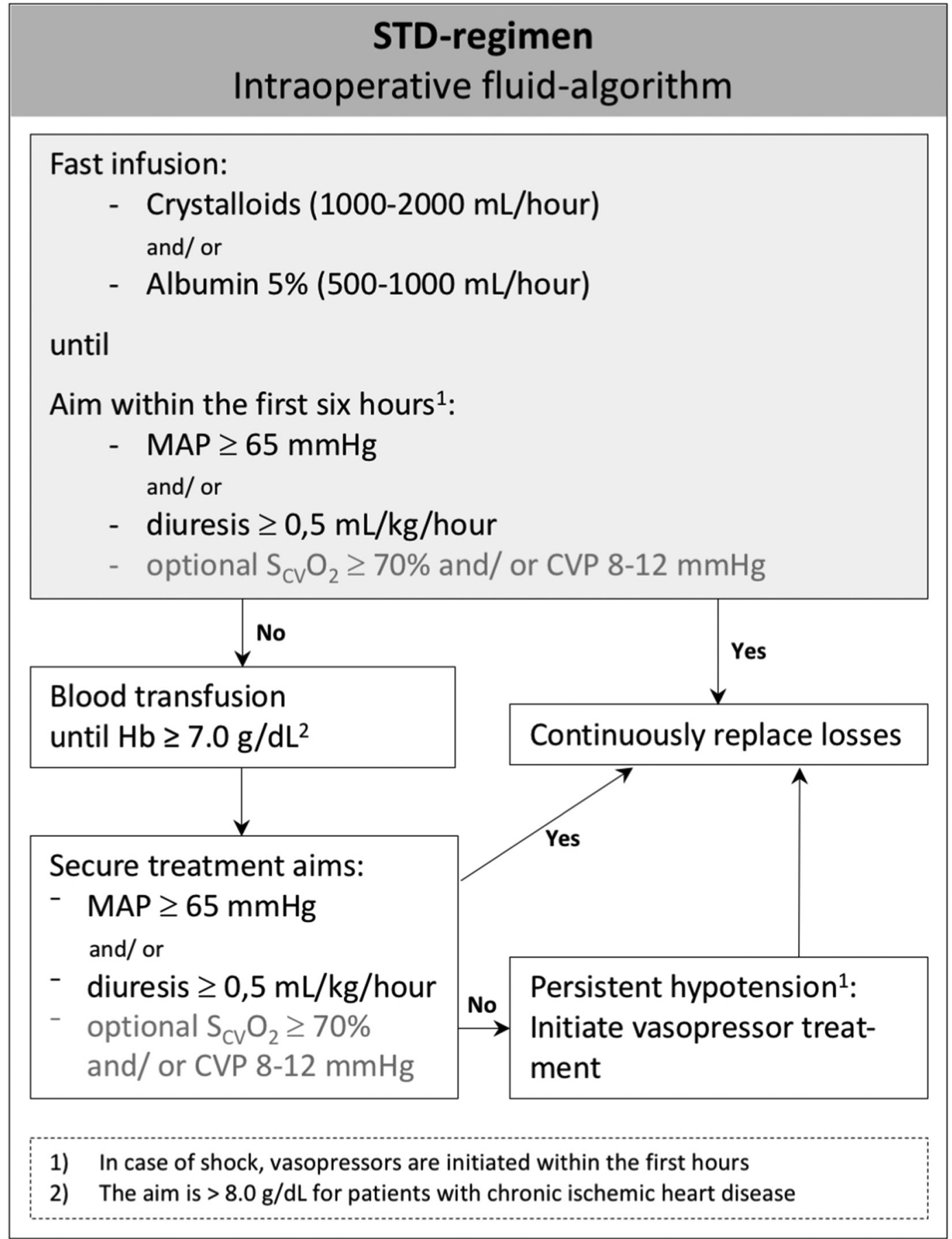

Figure 2 STD regimen. Intraoperative fluid algorithm. CVP, central vein pressure; Hb, haemoglobin; MAP, mean arterial pressure; $\mathrm{S}_{\mathrm{CV}} \mathrm{O}_{2}$, central venous oxygen saturation; STD, standard group.

increases more than $2 \mathrm{~kg}$. Prolonged intestinal paralysis might cause a net body weight gain of some kilograms and should not result in furosemide administration. Thus, clinical assessment alongside the data from fluid charts and body weight conditions the fluid replacement.

In the STD group, Ringers solution, saline $0.9 \%$ or albumin $5 \%$ are given intraoperatively to secure MAP $>65 \mathrm{~mm} \mathrm{Hg}$ and/or hourly diuresis $>0.5 \mathrm{~mL} / \mathrm{kg} /$ hour in accordance with the flowchart illustrated on figure 2. A central venous line is placed if the treating clinician finds it useful and CVP and $\mathrm{S}_{\mathrm{CV}} 0_{2}$ used to guide the fluid therapy. Vasopressors are used only if persistent hypotension (MAP <65 mm Hg) occurs.

Postoperative fluid therapy is given according to local routines aiming at acceptable MAP $>65 \mathrm{~mm} \mathrm{Hg}, \mathrm{HR}<100$ / min and diuresis $>1 \mathrm{~mL} / \mathrm{kg} /$ hour.

In both groups, postoperative analgesia is achieved with paracetamol, non-steroid anti-inflammatory drugs and/ or opioids according to guidelines, besides epidural analgesia in case of laparotomy. Early mobilisation is endorsed by physiotherapist. Ventricular retention (failure to tolerate per oral intake) is treated with a gastric tube. 
Metoclopramide or ondansetron is offered as antiemetic on demand.

The allocated treatment will be discontinued in all cases of a reoperation. The incidence will be registered as a part of the primary outcome. There is no other criteria for discontinuing the treatment in either group. Postoperative circulatory instability with a need for immediate care for example, septic shock is handled by the treating physician according to regional guidelines and overruling the allocated treatment. However, when the urgent phase has ended, the treatment returns to the allocated regimen and the episode is registered as an outcome.

Overall protocol adherence is secured by the project anaesthetist intraoperatively and postoperatively by the local investigator at the surgical ward. Individually training of selected trial physicians at the surgical and aesthetic wards was completed before study initiation at each involved hospital to secure protocol adherence. Formal presentation of the study was given to all staff at the intensive care unit, surgical and anaesthetic wards. Pocket cards with flowcharts were distributed. During the study period, continuous education is given to nurses and physicians in the teams responsible for the urgent surgical patients.

We use a detailed monitoring plan to evaluate protocol compliance. Regular reviewing of intraoperative and postoperative treating-related data at each hospital is conducted by the project leader as well as an independent data monitoring committee 'the unit of Good Clinical Practice', which assesses all phases of the GAS-ART trial. For example, intraoperatively one initial dose of human albumin and less than $2 \mathrm{~mL} / \mathrm{kg}$ /hour of maintenance fluid is expected in the GDT group and infusion of more than $2000 \mathrm{~mL}$ crystalloids in the STD group. Monitoring reports are acquired on a regular basis throughout the entire study period. Areas with potential protocol deviations are corrected continuously.

\section{Participant timeline}

See figure 3 for the GAS-ART timeline.

\section{Patient and public involvement}

Patients or public were not involved in the development of the research question or outcome measures. Only medically trained physicians carry out the patient recruitment and management in the study. A trial physician gives written and oral information on positive and negative aspects of the intervention to the patient. Simultaneously, information on the final study results is offered to all participating patients on request.

\section{OUTCOME MEASURES}

All outcome measures are registered 30 days postsurgical. The primary outcome is registered 90 days postsurgical. Table 1 lists the outcome measures.

\section{Primary composite outcome}

- All-cause mortality

- Unplanned reoperations
- Any unplanned laparotomy or laparoscopy

- Life-threatening bleeding complications

- For example, cerebral haemorrhage, disseminated intravascular coagulation or other bleeding leading to medical, surgical or endoscopic intervention

- Life-threatening thromboembolic events

- For example, acute myocardial infarction, stroke, pulmonary or intestinal emboli/thrombosis.

- Respiratory insufficiency

- Demanding mechanical respiratory support including non-invasive ventilation

- Cardiac arrest (survived)

- Renal failure

- Demanding dialysis

\section{Secondary outcome measures}

The secondary outcome measures are minor complications different from the primary composite outcome and specified time spans.

- Minor complications: A complication with a need for medical or surgical treatment.

- Timespan in the operating room, at the recovery room, at the intensive care unit, with mechanical respiratory support and until death.

- Postoperative days with dialysis.

- Length of hospital stay.

\section{Sample size}

Database inventory from Denmark shows a postoperative mortality rate of $15.7 \%$ after acute colonic surgery and one of $18.2 \%$ after operation for perforated ulcer. ${ }^{45}$ The most ill patients are incapable of giving informed consent, thus excluded. However, the frequency of major complications is expected to be high in the population. We estimated the combined incidence of overall death and major complications to be $25 \%$. A previous study testing a similar intervention in elective major gastrointestinal surgery has shown a risk reduction of $50 \%^{8}$ and we estimated a reduction in the combined outcome to $12.5 \%$. We accepted a $5 \%$ risk of type 1 error and a power to detect a reduction of $80 \%$. The calculated sample size was 149 patients in each group. The variance is unlikely to follow a normal distribution and the number of patients needed was adjusted to 155 in each group-a total of 310 patients. We plan no interim analysis.

\section{Recruitment}

Teams of dedicated trial physicians at each hospital screen all adult patients with a need for urgent gastrointestinal surgery according to the eligibility criteria. Verbal and written study information is given prior to surgery by the surgeon or anaesthetist and written informed consent is obtained before randomisation. With a planned inclusion period of 2 to 3 years, we chose to initiate the trial in five hospitals. If the inclusion rate is unexpectedly low, additional physicians will be trained to perform the GDT intervention. 


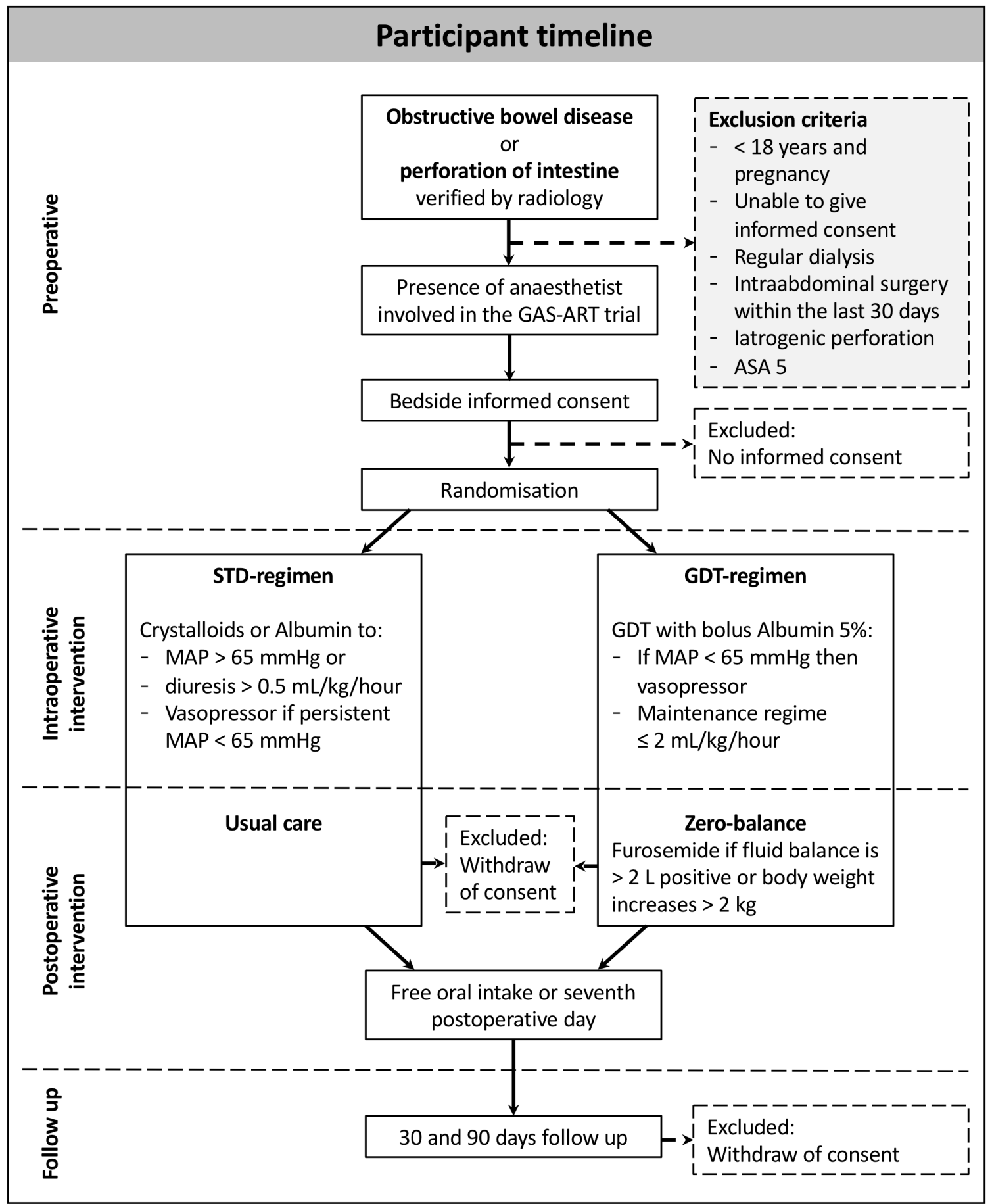

Figure 3 Participant timeline. ASA, American Society of Anaesthesiologists; GAS-ART, GAstrointestinal Surgery Study protocol for A Randomised multicentre Trial; GDT, goal-directed fluid therapy; MAP, mean arterial pressure; STD, standard. 
Table 1 Outcome measures

\section{Complication}

\begin{tabular}{ll}
\hline Abdominal & Superficial wound rupture \\
& Superficial wound haematoma \\
& Superficial wound infection
\end{tabular}

Wound infection and fascial defect

Facial rupture

Anastomosis leakage

Separation of stoma

Re-perforation

Peritonitis

Intra-abdominal abscess

Obstructive bowel disease

Prolonged paralysis of intestine

Gastrointestinal bleeding

Reoperation

Infectious Sepsis

Pneumonia

Cystitis

Other

$\begin{array}{ll}\text { Cardiopulmonic } & \text { Atrial arrhythmia } \\ & \text { Ventricular arrhythmia } \\ & \text { Acute myocardial infarction } \\ & \text { Cardiac arrest }\end{array}$

Exudation to the pleural cavity

Pulmonary congestion

Pulmonary oedema

Mechanical respiratory support

Acute respiratory distress

syndrome (ARDS)

Other

\begin{tabular}{ll} 
Thromboembolic & $\begin{array}{l}\text { Pulmonary embolism } \\
\text { Deep venous thrombosis } \\
\end{array}$ \\
\hline Other \\
\hline Renal & $\begin{array}{l}\text { Renal failure } \\
\text { Other }\end{array}$ \\
\hline $\begin{array}{l}\text { Central nervous } \\
\text { system }\end{array}$ & Stroke or cerebral haemorrhage
\end{tabular}

Delirium/psychosis

Other

\section{Definition}

Conservative or surgical treatment

Observed by a physician

Wound rupture, a need for removal of infected tissue or medical treatment

A need for surgical cleavage or removal of infected tissue with fascial defect

Spontaneously fascial rupture

Symptomatic and requiring treatment

Cutaneous and subcutaneous defect

A need for relaparotomy

Debut intraoperatively or postoperatively

Suspected radiologically with a need for medical or surgical treatment

A need for relaparotomy

7 days without flatus or faeces

A need for surgical or endoscopic treatment

Other unplanned intra-abdominal reoperations

Worsening postoperatively, debut intraoperatively or postoperatively. Graded according to sepsis-2 definitions ${ }^{43}$

Radiological documentation and one clinical sign: fever, leucocytosis, coughing or crepitus

Symptomatic and documented bacteriuria

With a need for medical or surgical intervention

Verified by ECG and a need for treatment

Verified by ECG and a need for treatment

ECG pathology and elevated cardiac enzymes

Diagnosed by a physician with or without successfully resuscitation

Verified by radiology

Suspected clinically with bilateral crepitus and positive effect of diuretic treatment

Radiographic suspicion and a need for intensive care

A need for intubation or continuous non-invasive ventilation

ARDS according to the Berlin definition ${ }^{44}$

With a need for medical or surgical intervention

Verified by scintigraphy or CT scan

Verified by radiology

With a need for medical or surgical intervention

A need for dialysis

With a need for medical or surgical intervention

Neurological symptoms and relevant radiology or diagnosed by neurologist

Deficiency in orientation, level of consciousness, cognition and/or psychosis

With a need for medical or surgical intervention 


\section{ASSIGNMENT OF INTERVENTION}

\section{Allocation}

We use a computer-generated block randomisation offered by OPEN-Randomise (Odense Patient data Explorative Network). We stratify by each hospital, and between obstructive bowel disease and perforation of the gastrointestinal tract. The study group of trial physicians is given access to OPEN-Randomise by username and password. The allocation sequence is concealed for all members of the study group. Trial physicians document the randomisation identifier number and allocation in the patient record and the case report form.

\section{Blinding}

The fluid therapy given in the GAS-ART trial includes the intraoperative and postoperative period at shifting departments involving many physicians and nurses. Additionally, clinical signs of hypovolaemia or hypervolaemia, for example, blood pressure, pulse, venous saturation, exudation to the pulmonic cavity, pulmonic oedema, subcutaneous oedema and diuresis are almost impossible to blind for the investigators. Thus, we could not find an effective way to blind the fluid therapy given in this trial. However, when the trial is completed, we conduct a blinded assessment of the primary outcome based on electronical patient records.

\section{DATA COLLECTION, MANAGEMENT AND ANALYSIS}

Intraoperative recording of fluid charts, blood pressure, HR and vasopressor use is collected by the trial physician. SV is recorded in the GDT group. In the postoperative phase, all enrolled patients are seen daily by a doctor on rounds who ensures protocol adherence. Data collection is in the electronical patient record, identified by the Danish personal registration number (CPR—det Centrale PersonRegister) including laboratory tests, radiology findings, microbiological results and other paraclinical results. Fluid charge and body weight are collected on paper forms. All patients will be Acute Physiology And Chronic Health Evaluation (APACHE II) scored the first day after surgery and patients admitted to the intensive care unit will be SOFA () scored daily. Follow-up is by the local investigator on postoperative days 30 and 90 either by telephone interview or by outpatient visit. Data transfer to the case report forms is secured only by the local investigator and trial physicians at each hospital to promote data quality and reviewed by the project leader. Additionally, registration of the primary outcome will be done by two independent and blinded accessors reading the patient records from all participating patients, blinded for information on the given fluid therapy, body weight measurements and allocation.

Case report forms and patient records will be stored at Holbaek Hospital in a secured locker. A database on the safe network of Holbaek Hospital is used for data entry securing confidentiality and allowing range checks for data validation. The steering committee will have access to the final trial dataset. Trial physicians can apply for access to the database. Local investigators will have access to trial data at their respective hospital.

\section{Statistical methods and analysis}

All data will be tested for normality and parametric statistics used for normal distributed data, otherwise non-parametric statistics are used. $\chi^{2}$ test will be used for binominal data, Fischer's exact test when expected values are below 5. Risk will be calculated when relevant.

The primary composite outcome will be reported as 'intention to treat'. The result will be presented as an entity and separately for explorative reasons. If more than 20 patients are excluded after the randomisation, a 'per-protocol' analysis will be added. If baseline characteristics are skewed, the effect will be assessed using a multiple logistic regression model and the adjusted as well as the unadjusted analysis of the primary outcome will be presented. If more than $10 \%$ of the intraoperative data are missing, a worst-case and a best-case scenario will be presented. If the results are inconclusive, multiple imputations will be performed. Results will be presented two-tailed and a p-value $<0.05$ is considered significant.

The secondary outcomes will be analysed as 'intention to treat' and 'per protocol' if more than 20 patients are excluded after randomisation. Sequence for analysis is as follows: minor complications, specified time spans, physiological data and finally data from blood samples.

Subsequently, subgroup analysis of the hypothesis presented in the Introduction section will be done. We plan analysis by centre and type of operation (laparoscopic vs laparotomy) to detect potential differences between hospitals and the two treatments. The incidence and severity of sepsis is likely to differ between patients with gastrointestinal perforation or obstructive bowel disease and data analysed separately focusing on number of organ failures. The results will highlight trends for further investigation as the number of participants is inconsequential.

\section{DATA MONITORING}

The GAS-ART trial will be conducted in adherence to International Council for Harmonisation-Good Clinical Practice (ICH-GCP) guidelines and the Declaration of Helsinki with data collection on paper case report forms. The GAS-ART trial is monitored by the independent units of Good Clinical Practice from Odense and Copenhagen securing an external monitoring of more than $10 \%$ of the completed case rapport forms and concordance with the ICH-GCP guidelines.

Four specified serious adverse reactions (SARs) as well as suspected unexpected serious adverse reactions (SUSARs) will be reported according to regulations from the unit of Good Clinical Practice and the Danish Health and Medicines Authority when suspected directly related to the infusion of saline, albumin or 
Ringer's solution: acute anaphylactic reaction, hypernatraemia (s-Na $>155 \mathrm{mmol} / \mathrm{L})$, central pontine myelinolysis and seizures. The units hold the ability to abort the study if deemed necessary. The presence of SAR or SUSAR does not exclude the patient from the study since continuous fluid replacement therapy is unavoidable.

Patients withdrawing their consent are excluded from further intervention and data collection. Cases are expected to be few because the treatment and data collection rely on the healthcare professionals and deviation from usual practice is of little inconvenience for the patient. Patients withdrawing their consent will be replaced. Due to safety regulations, any observed SAR and SUSAR will be registered for all included patients.

\section{ETHICS AND DISSEMINATION}

The trial was approved by the Danish Scientific Ethics Committee and the Danish Data Protection Agency (REG-18-2015) before patient enrolment. The study is classified as a drug trial by the Danish Medicines Agency (2014 1213 19). The trial is registered at the European Clinical Trials Database.

We identify no additional risk for the patients enrolled in the GAS-ART trial compared with usual practice. Arterial lines are generally used in patients with a need for urgent gastrointestinal surgery, and no additional invasive procedures will be applied.

\section{Dissemination plan}

We plan to publish study results in an international peer-reviewed journal. Negative as well as positive results will be published. Authors will have to meet the principles of the Vancouver Declaration.

The results will be presented at both national and international conferences of relevance. A letter will inform the participants about the study results when requested. Furthermore, the results will be presented at all involved hospitals and participating wards.

\section{Trial status}

Patient inclusion was initiated in August 2015 at Holbaek University Hospital. The additional four centres were consecutively introduced. By February 2016, the five planned centres had begun patient inclusion. We expect that patient inclusion will increase as study algorithms gradually become integrated in the daily routines at each centre. On 23 February 2018, 243 patients were included.

\section{DISCUSSION}

The GAS-ART trial is a randomised clinical trial testing the effect of two distinct fluid regimens on postoperative complications and death following urgent gastrointestinal surgery. Through the last 30 years, the effect of GDT optimisation has been tested on selected surgical patients, including high-risk patients. However, only a few studies have included emergency gastrointestinal surgical patients, and no trial has specifically targeted patients undergoing urgent surgery for obstructive bowel disease or gastrointestinal perforation. ${ }^{26-30}$ Patients scheduled for urgent gastrointestinal surgery are often fluid deranged and preseptic at hospital admission, thus deviating markedly from the patients scheduled for planned surgery, and accordingly the mortality and complication rates are pronounced. ${ }^{631}$ Studies of care bundles in emergency gastrointestinal surgery and meta-analysis suggest that high-risk surgical patients benefit from GDT optimisation. ${ }^{32-34}$ The results has led to a widespread use of GDT algorithms within urgent gastrointestinal surgery. In opposition, a Cochrane review and a recent meta-analysis found no evidence to support this general implementation. $^{35} 36$

The GAS-ART study is designed in accordance with a firm scientific structure and holds several strengths. The computer-generated block randomisation is blinded for all investigators, even the project leader. Furthermore, the units of Good Clinical Practice audits all patients enrolled in the trial and a list of all potential study patients, all together minimising the chance of allocation bias. The patient group is easily identified in daily clinical practice using standard radiological actions for the diagnosis combined with limited exclusion criteria favouring consecutivity of patient enrolment and lessening the chance of selection bias. The treatment in both the STD group and the GDT group was taught thoroughly to a selected group of trial physicians, responsible for the execution of the trial at each hospital. Additionally, continuously individual and formal teaching by one project leader secured unified implementation of both treatment regimens to lower performance bias. The primary outcome is clear, patient relevant and per-protocol defined consisting of six severe complications and death, and two previously randomised clinical trials emphasises their applicability. ${ }^{87}$ The primary outcome markers demand medical or surgical treatment and will without exception be found in the patient's records. Therefore, we assume no missing data related to the primary outcome measures. Additionally, we plan a blinded assessment of the primary outcome to reduce detection bias. Data on fluid administration during surgery and at the postoperative care unit is routinely registered and we expect few missing data. Likewise, the secondary outcomes of minor complications call for treatment and few missing data are expected. In addition, the time periods of interest are all easily found in the patient journal.

The complexity of the study set-up, however, holds weaknesses that need to be addressed. The very nature of urgent surgery might impair enrolment of patients in the GAS-ART trial. First, operation should be performed as fast as possible, giving little time for information of the patient, little time for reflection and thus little time for informed consent. Second, the treating physicians and nurses focus on fast, often life-saving, treatment and stabilisation of the patient, which might compromise the time for study-related chores and limit patient enrolment. 
Third, limiting patient enrolment to selected anaesthetists might be challenging because of working shifts. Blinding of investigators and patients was not doable under the given circumstances, holding a potential of performance bias. Emphasis on treatment optimisation might be applied to patients in the GDT group, potentially affecting the outcome not only related to the two different fluid regimens. On the other hand, the overall training and focus on intraoperative and postoperative fluid optimisation might enhance the general treatment and unify the care in the STD group and GDT group, thus eliminating a hypothetical positive effect of either fluid regimen. Finally, the use of a composite outcome holds a defiance. The most essential shortcoming of a composite outcome is the interpretation of the results. The difference between death and a severe complication is infinite in most situations, but not possible to distinguish in the result. However, the outcome parameters in the present trial will be reported both separately and as an entity.

The choice of intervention fluids needs to be addressed. GDT optimisation is usually based on consecutive infusion of hydroxyethyl starch entities. However, two recent studies showed increased need for renal replacement therapy and death using hydroxyethyl starch for fluid resuscitation in patients admitted at the intensive care unit. In addition, a Cochrane review questions the use of hydroxyethyl starch in randomised trials. ${ }^{38-40}$ Use of crystalloids for GDT optimizations results in increased amounts of total intravenous fluid infusion. ${ }^{41}$ The excessive infusion of chloride-containing fluids might cause hyperchloraemia which has been directly associated with increased 30-day mortality and length of hospital stay. ${ }^{42}$ With an aim to avoid excessive fluid administration and due to the disputes about hydroxyethyl starch, we chose to use albumin for GDT optimisation in the GDT group. Additionally, the treatment algorithm in the STD group includes albumin for resuscitation and hinders a potential treatment bias.

The GAS-ART trial provides strong imperative results with a markedly clinical potential. Urgent gastrointestinal surgery is common and optimisation of the perioperative fluid regimens holds the potential to reduce postoperative death, complications and readmissions.

\begin{abstract}
Acknowledgements We are thankful for the local investigators and their enthusiasm. Thanks to Nicolas Storm (MD, staff specialist, surgical department, Herlev), Erik Zimmermann-Nielsen (MD, chief surgeon, surgical department, Odense), Søren Trap Andersen (MD, chief physician, anaesthetic department, Svendborg) and Pernille Tibæk (MD, surgical department, Slagelse) who have contributed substantially to secure implementation and the ongoing enrolment of patients in the GAS-ART trial. OPEN-Randomise (Odense Patient data Explorative Network) is responsible for the computer-generated randomisation.
\end{abstract}

Contributors BB: Developed the idea, searched the literature, drafted the protocol, conducted the power calculation, framed the intervention and the control treatment, planned the study, collected the data, planned the analysis and interpretation, revised and approved the final protocol. Raised the funds. AMM: Revised the intervention and the control treatment, planned the study, planned the analysis and interpretation, revised and approved the final protocol. AWV: Drafted the present manuscript, refined and revised the drafted protocol, registered the trial at EudraCT, obtaining legislative and ethical approvals, planned the study, revised the intervention, collected the data, revised the analysis and interpretation, revised and approved the final protocol. Responsible for initiating and conducting the trial. Raised the funds. AAA: Revised the drafted protocol, planned the study and intervention, collected the data, revised the analysis and interpretation, revised and approved the final protocol. Responsible for conducting and completion of the trial. Raised the funds. The steering committee consists of BB, AMM, AWV and AAA besides a local investigator from each participating hospital and is responsible for the inclusion and randomisation of patients, the protocol adherence, the data collection and the daily monitoring. The steering committee will critically read the final report and thereby have final authority over the report submitted for publication. The units of Good Clinical Practice monitor the trial at all centres participating.

Funding The study is funded by Department of Surgery, Holbaek University Hospital; the Trans-regional Fund between Zealand Region and The Region of South Denmark; the independent research unit of Zealand Region in Denmark; Axel Muusfeldts fund; Department of Anaesthesiology and Intensive Care, Holbaek University Hospital; Department of Anaesthesiology and Intensive Care, Herlev University Hospital; Department of Anaesthesiology and Intensive Care, Odense University Hospital. The author BB is the sponsor of the GAS-ART trial and the funders have no role in planning, conducting, collection of data or analysis in the trial. The financial support to the project is unrestrained.

Competing interests None declared.

Patient consent Not required.

Ethics approval The trial was approved by the Danish Scientific Ethics Committee (J: SJ-436).

Provenance and peer review Not commissioned; externally peer reviewed.

Open access This is an open access article distributed in accordance with the Creative Commons Attribution Non Commercial (CC BY-NC 4.0) license, which permits others to distribute, remix, adapt, build upon this work non-commercially, and license their derivative works on different terms, provided the original work is properly cited, appropriate credit is given, any changes made indicated, and the use is non-commercial. See: http://creativecommons.org/licenses/by-nc/4.0/.

\section{REFERENCES}

1. Ingraham AM, Cohen ME, Bilimoria KY, et al. Comparison of 30-day outcomes after emergency general surgery procedures: potential for targeted improvement. Surgery 2010;148:217-38.

2. Saunders DI, Murray D, Pichel AC, et al. Variations in mortality after emergency laparotomy: the first report of the UK Emergency Laparotomy Network. Br J Anaesth 2012;109:368-75.

3. Barrow E, Anderson ID, Varley S, et al. Current UK practice in emergency laparotomy. Ann R Coll Surg Engl 2013;95:599-603.

4. Jørgensen HS. Danish Clinical Register of Emergency surgery for Peptic Ulcer. Danish Clinical Register of Emergency Surgery for Peptic Ulcer. Annual report 2015. Akut Kir Database 2016.

5. Vester-Andersen M, Waldau T, Wetterslev J, et al. Randomized multicentre feasibility trial of intermediate care versus standard ward care after emergency abdominal surgery (InCare trial). Br J Surg 2015;102:619-29.

6. Pearse RM, Harrison DA, James P, et al. Identification and characterisation of the high-risk surgical population in the United Kingdom. Crit Care 2006;10:R81.

7. Vester-Andersen M, Lundstrøm LH, Waldau T, et al. High mortality following emergency gastrointestinal surgery: a cohort study. Eur $J$ Anaesthesiol 2013;30:4-5.

8. Brandstrup B, Tønnesen H, Beier-Holgersen R, et al. Effects of intravenous fluid restriction on postoperative complications: comparison of two perioperative fluid regimens: a randomized assessor-blinded multicenter trial. Ann Surg 2003;238:641-8.

9. McArdle GT, McAuley DF, McKinley A, et al. Preliminary results of a prospective randomized trial of restrictive versus standard fluid regime in elective open abdominal aortic aneurysm repair. Ann Surg 2009;250:28-34.

10. Gao T, Li N, Zhang JJ, et al. Restricted intravenous fluid regimen reduces the rate of postoperative complications and alters immunological activity of elderly patients operated for abdominal cancer: a randomized prospective clinical trail. World J Surg 2012;36:993-1002.

11. Salzwedel C, Puig J, Carstens A, et al. Perioperative goaldirected hemodynamic therapy based on radial arterial pulse pressure variation and continuous cardiac index trending reduces postoperative complications after major abdominal surgery: a multicenter, prospective, randomized study. Crit Care 2013;17:R191. 
12. Benes J, Chytra I, Altmann P, et al. Intraoperative fluid optimization using stroke volume variation in high risk surgical patients: results of prospective randomized study. Crit Care 2010;14:R118.

13. Mayer J, Boldt J, Mengistu AM, et al. Goal-directed intraoperative therapy based on autocalibrated arterial pressure waveform analysis reduces hospital stay in high-risk surgical patients: a randomized, controlled trial. Crit Care 2010;14:R18.

14. Bjerre CC, Holte K. [Perioperative fluid therapy in perforated ulcers]. Ugeskr Laeger 2009;171:1488-91.

15. Lilot M, Ehrenfeld JM, Lee C, et al. Variability in practice and factors predictive of total crystalloid administration during abdominal surgery: retrospective two-centre analysis. $\mathrm{Br} \mathrm{J}$ Anaesth 2015;114:767-76.

16. Nisanevich V, Felsenstein I, Almogy G, et al. Effect of intraoperative fluid management on outcome after intraabdominal surgery. Anesthesiology 2005;103:25-32.

17. Piljic D, Petricevic M, Piljic D, et al. Restrictive versus standard fluid regimen in elective minilaparotomy abdominal aortic repairprospective randomized controlled trial. Thorac Cardiovasc Surg 2016;64:296-303.

18. Noblett SE, Snowden CP, Shenton BK, et al. Randomized clinica trial assessing the effect of Doppler-optimized fluid management on outcome after elective colorectal resection. Br J Surg 2006;93:1069-76.

19. Wakeling HG, McFall MR, Jenkins CS, et al. Intraoperative oesophageal Doppler guided fluid management shortens postoperative hospital stay after major bowel surgery. $\mathrm{Br} J$ Anaesth 2005;95:634-42

20. Phan TD, Ismail H, Heriot AG, et al. Improving perioperative outcomes: fluid optimization with the esophageal Doppler monitor, a metaanalysis and review. J Am Coll Surg 2008;207:935-41.

21. Rahbari NN, Zimmermann JB, Schmidt T, et al. Meta-analysis of standard, restrictive and supplemental fluid administration in colorectal surgery. Br J Surg 2009;96:331-41.

22. Møller MH, Adamsen S, Thomsen RW, et al. Multicentre trial of a perioperative protocol to reduce mortality in patients with peptic ulcer perforation. Br J Surg 2011;98:802-10.

23. Rivers E, Nguyen B, Havstad S, et al. Early goal-directed therapy in the treatment of severe sepsis and septic shock. N Engl J Med 2001;345:1368-77.

24. Chan AW, Tetzlaff JM, Altman DG, et al. SPIRIT 2013 statement: defining standard protocol items for clinical trials. Ann Intern Med 2013;158:200.

25. Jørgensen HS. on behalf of the Danish Akut kirurgi Database. Danish national database on colonorectal cancer. Annual repport 2014. 2014:01-182

26. Shoemaker WC, Appel PL, Kram HB, et al. Prospective trial of supranormal values of survivors as therapeutic goals in high-risk surgical patients. Chest 1988;94:1176-86.

27. Sandham JD, Hull RD, Brant RF, et al. A randomized, controlled trial of the use of pulmonary-artery catheters in high-risk surgical patients. N Engl J Med 2003;348:5-14.

28. Pearse R, Dawson D, Fawcett J, et al. Early goal-directed therapy after major surgery reduces complications and duration of hospital stay. A randomised, controlled trial [ISRCTN38797445]. Crit Care 2005;9:R687-93.

29. Pearse RM, Harrison DA, MacDonald N, et al. Effect of a perioperative, cardiac output-guided hemodynamic therapy algorithm on outcomes following major gastrointestinal surgery: a randomized clinical trial and systematic review. JAMA 2014;311:2181-90.

30. Boyd O, Grounds RM, Bennett ED. A randomized clinical trial of the effect of deliberate perioperative increase of oxygen delivery on mortality in high-risk surgical patients. JAMA 1993;270:2699-707.

31. Ozdemir BA, Sinha S, Karthikesalingam A, et al. Mortality of emergency general surgical patients and associations with hospital structures and processes. Br J Anaesth 2016;116:54-62.

32. Dalfino L, Giglio MT, Puntillo F, et al. Haemodynamic goal-directed therapy and postoperative infections: earlier is better. A systematic review and meta-analysis. Crit Care 2011;15:R154.

33. Huddart S, Peden CJ, Swart M, et al. Use of a pathway quality improvement care bundle to reduce mortality after emergency laparotomy. Br J Surg 2015;102:57-66.

34. Tengberg LT, Bay-Nielsen M, Bisgaard T, et al. Multidisciplinary perioperative protocol in patients undergoing acute high-risk abdominal surgery. Br J Surg 2017;104:463-71.

35. Grocott MP, Dushianthan A, Hamilton MA, et al. Perioperative increase in global blood flow to explicit defined goals and outcomes following surgery. Cochrane Database Syst Rev 2012;11:CD004082.

36. Rollins KE, Lobo DN. Intraoperative goal-directed fluid therapy in elective major abdominal surgery: a meta-analysis of randomized controlled trials. Ann Surg 2016;263:465-76.

37. Brandstrup B, Svendsen PE, Rasmussen M, et al. Which goal for fluid therapy during colorectal surgery is followed by the best outcome: near-maximal stroke volume or zero fluid balance? $\mathrm{Br}$ Anaesth 2012;109:191-9.

38. Perner A, Haase N, Guttormsen AB, et al. Hydroxyethyl starch $130 / 0.42$ versus Ringer's acetate in severe sepsis. N Engl J Med 2012;367:124-34.

39. Myburgh JA, Finfer S, Bellomo R, et al. Hydroxyethyl starch or saline for fluid resuscitation in intensive care. $N$ Engl J Med 2012;367:1901-11.

40. Perel P, Roberts I, Ker K. Colloids versus crystalloids for fluid resuscitation in critically ill patients. Cochrane Database Syst Rev 2013:CD000567.

41. Yates DR, Davies SJ, Milner HE, et al. Crystalloid or colloid for goal-directed fluid therapy in colorectal surgery. Br J Anaesth 2014;112:281-9.

42. McCluskey SA, Karkouti K, Wijeysundera D, et al. Hyperchloremia after noncardiac surgery is independently associated with increased morbidity and mortality: a propensity-matched cohort study. Anesth Analg 2013;117:412-21.

43. Levy MM, Fink MP, Marshall JC, et al. 2001 SCCM/ESICM/ACCP/ ATS/SIS International Sepsis Definitions Conference. Crit Care Med 2003;31:1250-6.

44. Ranieri VM, Rubenfeld GD, Thompson BT, et al. Acute respiratory distress syndrome: the Berlin Definition. JAMA 2012;307:2526-33. 